\title{
Using axon models to interpret electrodiagnostic nerve tests Karl Jensen ${ }^{1}$, Thu NA Luu ${ }^{1}$ and Kelvin E Jones*1,2
}

\author{
Address: ${ }^{1}$ Department Electrical and Computer Engineering, University of Alberta, Edmonton, AB, Canada, T6G $2 \mathrm{H} 9$ and ${ }^{2} \mathrm{Faculty}$ of Physical \\ Education \& Recreation, University of Alberta, Edmonton, AB, Canada, T6G 2H9 \\ Email: Kelvin E Jones* - kejones@ualberta.ca \\ * Corresponding author
}

from Seventeenth Annual Computational Neuroscience Meeting: CNS*2008

Portland, OR, USA. 19-24 July 2008

Published: II July 2008

BMC Neuroscience 2008, 9(SuppI I):P43 doi:I0.I I86/I47I-2202-9-SI-P43

This abstract is available from: http://www.biomedcentral.com/I47I-2202/9/SI/P43

(C) 2008 Jensen et al; licensee BioMed Central Ltd.

\section{Introduction}

Automated nerve excitability testing is a relatively new electrodiagnostic technique that became commercially available in 2007 [1]. The purpose of an excitability test is to infer the underlying membrane properties of the nerve in order to detect ion channel disorders in vivo. This is accomplished by using both supra- and sub-maximal conditioning stimuli of different amplitudes and latencies with respect to a test stimulus. The standard clinical protocol for motor axons includes four tests: 1) strengthduration; 2) recovery cycle; 3) threshold electrotonus; and 4) current-threshold. The interpretation of the four tests is complicated and mathematical models have been essential for explaining unexpected results [2]. This study's objectives were to: 1) compare two candidate motor axon models for interpreting nerve excitability studies; 2 ) perform a sensitivity analysis to establish correlations between membrane biophysics and clinical outcome measures; and 3) develop an optimization routine for fitting the models to experimental data.

\section{Methods}

A minimal model (node and internode) [2] was compared to a multicompartment model with detailed morphology [3]. All modeling and simulations were done using NEURON [4]. Both models have been previously published but a full sensitivity analysis and independent comparison on the complete set of clinical nerve excitability protocols has not been done. The minimal model has been fine-tuned to match excitability results whereas the multicompartment model was fit to intracellular recordings of myelinated rat axons. The sensitivity analysis was restricted to changes of $\pm 40 \%$ from default for active membrane properties.

\section{Results}

The minimal model provided a much better fit to data acquired from healthy control subjects as seen in Figure 1. The multicompartment model was especially poor at matching data from the tests $3 \& 4$ that evaluate ion channel function in the internodal region. The sensitivity analysis indicated that the current ion channel models in the multicompartment model are not capable of capturing the variation in the healthy control data. Based on the minimal model, much of the inter-individual variation in healthy controls arises from differences in resting membrane potential. We conclude that a hybrid model that uses the morphology of the multicompartment model and the ion channel kinetics from the minimal model will provide the most utility for interpreting nerve excitability tests. 

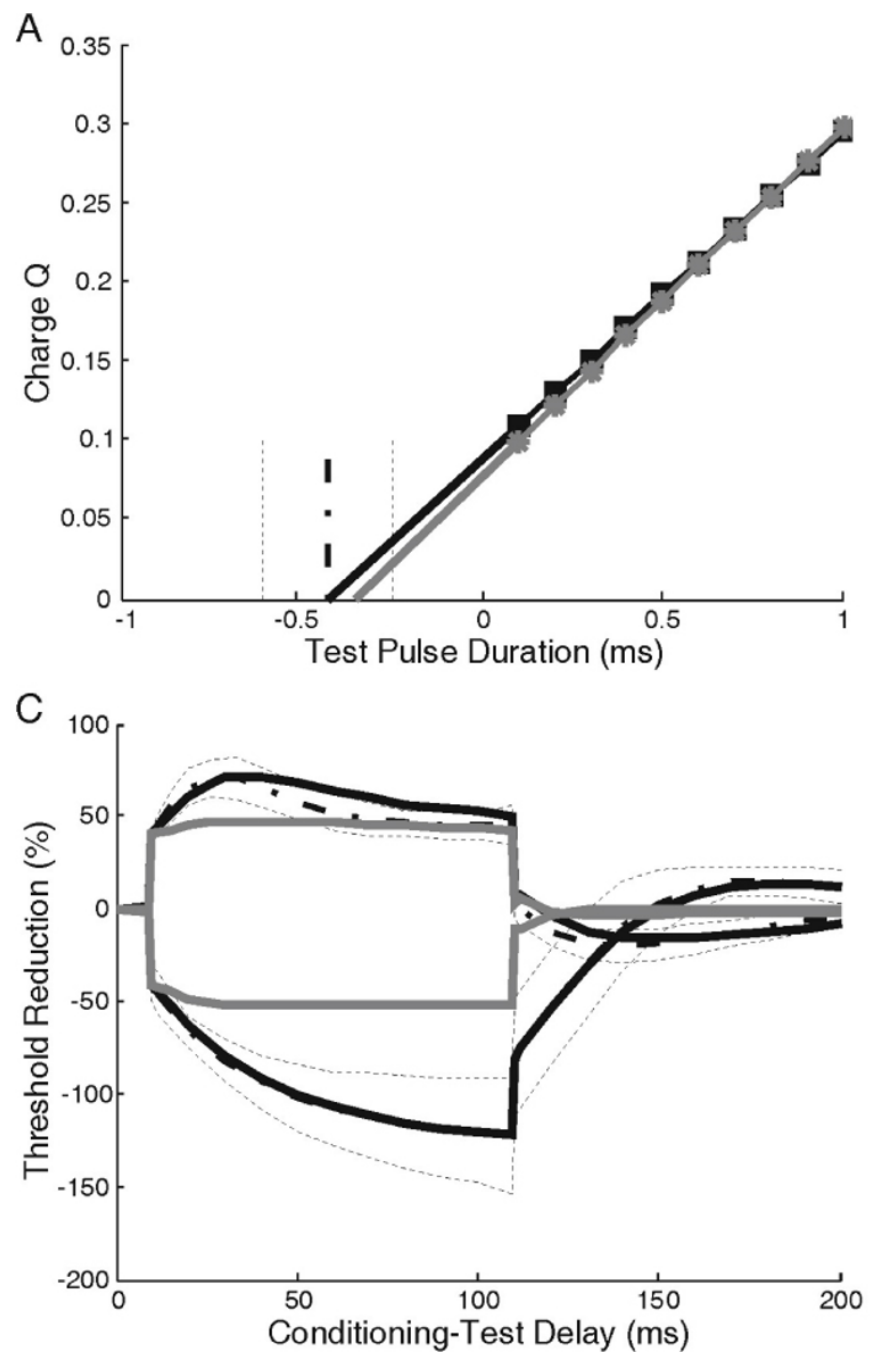

Figure I

Performance of minimal (black) versus multicompartment (grey) model on the four tests. Dot-dash line is the mean from healthy control data.

\section{Acknowledgements}

Supported by grants from AHFMR

\section{References}

I. Digitimer DS5 Isolated Bipolar Constant Current Stimulator and QtracW software [http://www.digitimer.com/clinical/ pstims.htm]

2. Kiernan MC, Isbister GK, Lin CS, Burke D, Bostock H: Acute tetrodotoxin-induced neurotoxicity after ingestion of puffer fish. Ann Neurol 2005, 57:339-348.

3. McIntyre CC, Richardson AG, Grill WM: Modeling the excitability of mammalian nerve fibers: influence of afterpotentials on the recovery cycle. J Neurophysiol 2002, 87:995-1006.

4. Carnevale NT, Hines ML: The NEURON Book Cambridge: Cambridge University Press; 2006.
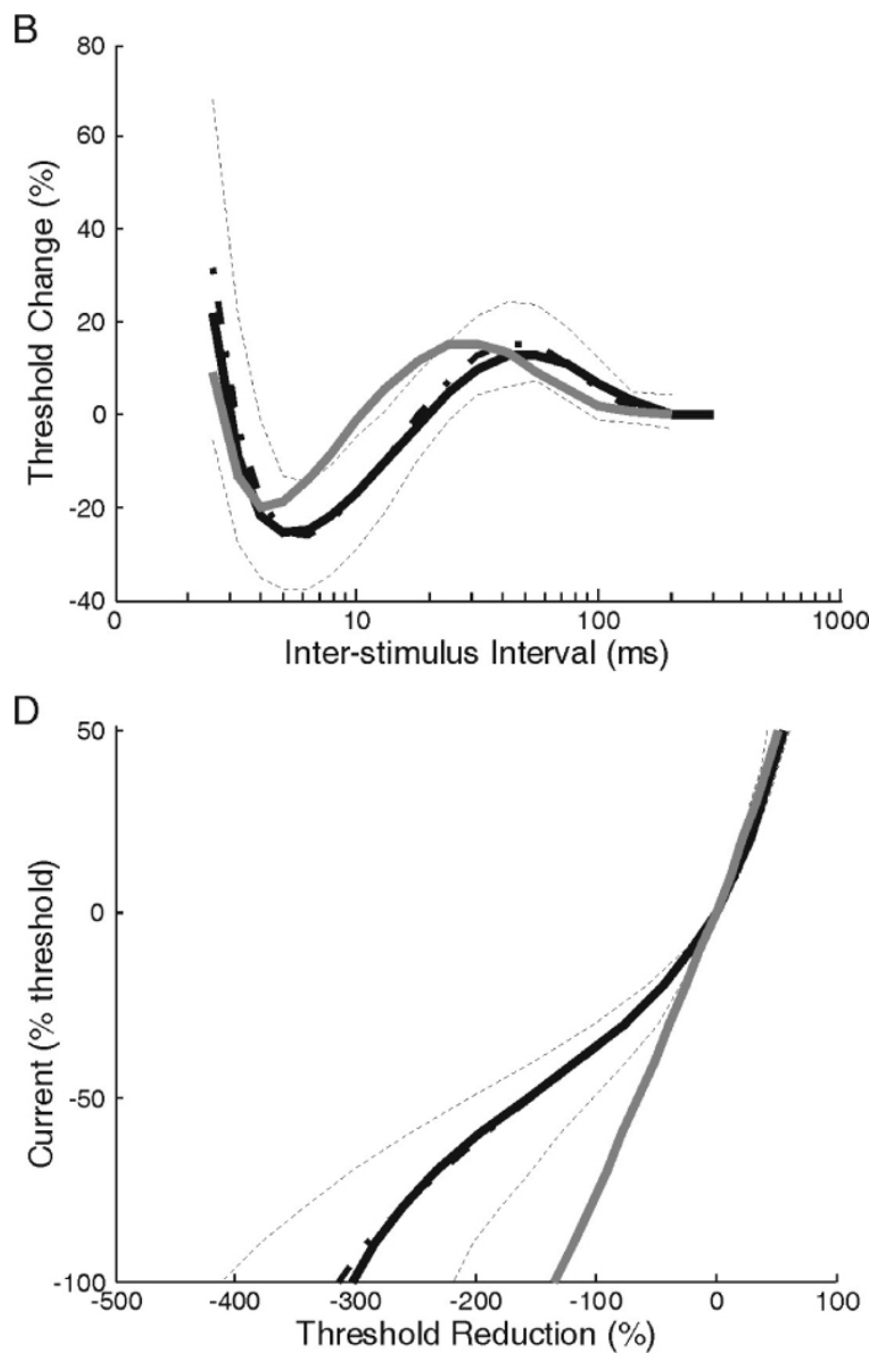